\title{
gamES: um Framework de Gamificação Baseado em Design Centrado no Usuário para o Aprendizado de Desenvolvimento de Software
}

\author{
Dariane Abich, Caciele Barbosa, Rafael Parizi \\ ${ }^{1}$ Grupo de Pesquisa Lardev \\ Instituto Federal Farroupilha - Campus São Borja \\ São Borja - Brazil \\ \{darianeabich, cacielebarbosa\}@gmail.com \\ rafael.parizieiffarroupilha.edu.br
}

\begin{abstract}
Software development is considered a challenge due to the constant changes in requirements, costs, and risks, the involvement of stakeholders, making it essential to develop solutions that meet the real needs of the user in this context. Thus, to achieve high-quality results, different skills are required, and teaching in this area is a complex activity. This article presents gamES, a framework aimed at teaching software development, integrating the User-Centered Design approach, and Gamification strategies. The methodological discourse was composed of two Design Thinking sessions, a prototyping stage, to gather and refine the requirements, and an evaluation stage for user validation. As a result, a list of requirements and a set of user evaluations had been produced, which demonstrated ease in understanding how to use the structure, in addition to identifying the elements of gamification.
\end{abstract}

Resumo. O desenvolvimento de software é considerado um desafio devido às constantes mudanças de requisitos, aos custos e riscos, ao envolvimento das partes interessadas, tornando essencial a elaboração de soluções que atendam às necessidades reais do usuário neste contexto. Assim, para alcançar resultados de alta qualidade são necessárias diferentes habilidades e ensinar, nessa área, é uma atividade complexa. Este artigo apresenta o gamES, um framework voltado ao ensino de desenvolvimento de software integrando a abordagem de Design Centrado no Usuário e estratégias de gamificação. O discorrer metodológico foi composto por duas sessões de Design Thinking, uma etapa de prototipação, para reunir e refinar os requisitos, e uma etapa de avaliação para validação de usuário. Como resultados, fora produzida uma lista de requisitos e um conjunto de avaliações de usuários, que demonstraram facilidade em entender como usar a estrutura, além da identificação dos elementos de gamificação.

\section{Introdução}

O desenvolvimento de software recebeu notoriedade, considerando a importância dos sistemas para a vida humana. As pessoas estão realizando ainda mais atividades diárias envolvendo softwares, seja para melhorar a qualidade de vida, mídia social, notícias ou saúde. Assim, aprender como desenvolver sistemas computacionais e atender às necessidades do usuário são desafios do campo da Engenharia de Software. 
Considerada uma atividade complexa por envolver um variado conjunto de requisitos, como conhecimento de diferentes linguagens de programação, arquitetura de software, segurança, banco de dados, entre outros [Pressman and Maxim 2014], o desenvolvimento de software é objeto de investigação. Azeem et al. [Azeem et al. 2019] menciona também que os desenvolvedores de software precisam atender a novos requisitos, corrigir falhas, lidar com falta de tempo e pressão das partes interessadas, conflitos de design e outros, causando problemas no processo que podem afetar a qualidade do software. Dessa forma, os cursos de desenvolvimento nessa área precisam ser eficazes para bem preparar os alunos para lidar com este contexto.

Alternativas de ensino têm sido apresentadas na literatura com foco em metodologias para criar software com qualidade, como, por exemplo, práticas orientadas ao aluno, que incluem Aprendizagem Baseada em Projeto (do Inglês Project Based Learning (PBL)) [Blumenfeld et al. 1991, Krajcik and Blumenfeld 2005], Aprendizado Ativo [Felder and Brent 2009] e Gamificação [Dicheva et al. 2015]. Porém, considerando as abordagens de Design Centrado no Usuário (UCD) para desenvolvimento de software [Kiunsi and Ferwerda 2019], o uso das estratégias de ensino precisa ser integrado ao Design Thinking (DT) [Vianna 2012] ou Lean Inception [Caroli 2018], por exemplo, a fim de alcançar soluções mais assertivas.

Neste sentido, as contribuições deste trabalho em andamento são: (i) integrar a abordagem de ensino de desenvolvimento de software através da gamificação e UCD usando o DT; (ii) apresentar gamES, uma estrutura para conduzir o processo de ensino de desenvolvimento de software usando conceitos de gamificação. A proposta, portanto, visa fomentar o raciocínio colaborativo, inovador e criativo, intermediado pelo DT. Além disso, busca-se fortalecer o ensino e aprendizagem de desenvolvimento de software através da participação ativa dos estudantes, estimulados através dos conceitos da Gamificação. Contudo, dado o andamento do trabalho, ainda não foram realizados testes com estudantes, os quais serão tratados em trabalhos futuros.

O artigo está estruturado da seguinte forma: Seção 2 apresenta um histórico e trabalhos relacionados. Na Seção 3, é mostrada a abordagem metodológica, enquanto na Seção 4 relata-se sobre a avaliação inicial do gamES. Finalmente, na Seção 5, estão descritas as considerações finais, os riscos à validade e os trabalhos futuros.

\section{Trabalhos relacionados}

A gamificação é definida como o uso de elementos de jogo e técnicas de design em contextos que não são de jogo [Werbach and Hunter 2015], ou seja, que não têm o intuito restrito ao entretenimento. Além disso, ela pode ser entendida como um processo de melhoria de um serviço através de reconhecimento de experiências de formas lúdicas, para apoiar a geração de valor ao usuário [Huotari and Hamari 2017].

Considerando as metodologias de ensino voltadas para o desenvolvimento de software, a gamificação têm sido utilizada em diferentes práticas. Brum e Cruz [Brum and da Cruz 2017] relatam a experiência do uso da gamificação no ensino de robótica, na qual foi possível verificar um avanço significativo no desempenho das aulas. Tomisaki [Tomisaki et al. 2016] expõe o seu relato no ensino de gerenciamento de projetos, usando um jogo de cartas que resultou na motivação dos alunos, impulsionando a performance acadêmica de maneira lúdica e eficaz. 
Ferreira e Oliveira [Ferreira-Oliveira et al. 2017] propõe um protótipo para a aplicação da gamificação como solução para problemas com o aprendizado de programação. O estudo de autoria de Poffo et al. [Poffo et al. 2017] mostra que a gamificação permite motivação intrínseca, apresentando a análise da relação entre motivação e o efeito no aprendizado. Simões et al. [Simões et al. 2013] considera que essa abordagem precisa ser apoiada por atividades como (a) fornecer experiências diferentes; (b) incluir ciclos rápidos de feedback; (c) aumentar a dificuldade das tarefas de acordo com a capacidade dos alunos; (d) dividir tarefas complexas por outras menores; (e) incluir erros como parte do processo de aprendizagem.

Outro campo que recebeu um aumento de atenção é o Design Centrado no Usuário, o qual coloca os usuários no centro do processo, considerando suas necessidades como os principais conceitos. Nesse ambiente, o uso de métodos como o Design Thinking visa estimular a inovação, transformar organizações e sociedades através da aplicação de suas técnicas. Para isso, é importante entender o papel fundamental do design e seus efeitos através do exercício do pensamento de forma multidisciplinar, a fim de delinear o campo do design e suas relações com negócios, gestão, inovação [Desconsi 2012].

Freitas et al. [Freitas et al. 2013] descrevem DT como um conjunto de técnicas e ferramentas centradas no usuário para produzir, analítica e criativamente, soluções para desafios reais. DT surge, então, como um conjunto de práticas inspiradas no design para resolver problemas e desenvolver soluções, usando empatia, criatividade e racionalidade para atender às necessidades dos usuários [Weigel 2015].

\section{Materiais e Métodos}

Este trabalho apresenta o gamES, uma ferramenta que combina gamificação e Design Thinking para ajudar no processo de aprendizado de desenvolvimento de software. A integração entre gamificação e DT busca envolver os alunos nas atividades fornecendo suporte ao desenvolvimento de software através de uma metodologia estruturada. O gamES utiliza DT como o método para praticar UCD, ou seja, os participantes são conduzidos pela ferramenta para executar atividades baseadas em DT, visando, ao final, produzir uma proposta de software que resolva um problema específico ou um problema da sociedade.

Para descobrir os principais recursos do gamES, um processo metodológico foi realizado com base nas seguintes etapas: (i) sessão dupla de Design Thinking: para conhecer o contexto, as necessidades dos usuários com relação ao aprendizado de desenvolvimento de software sob uma visão multidisciplinar; (ii) prototipação: para gerar o primeiro modelo de software da ferramenta; e (iii) validação de usuário: para, por meio de entrevistas, validar a solução proposta. A Figura 1 mostra as etapas da metodologia e os detalhes de cada uma delas.

\subsection{Sessão dupla do Design Thinking}

Inicialmente foram realizadas atividades de DT adaptadas com base no guia do Instituto Hasso Plattner [Hasso Plattner Institute 2010]. Essa abordagem de DT fornece recursos para envolver as partes interessadas em uma reflexão sobre a problemática, perguntandolhes sobre suas necessidades durante uma hora. Para tanto, a abordagem de DT foi organizada em duas sessões com seis participantes em cada uma. 


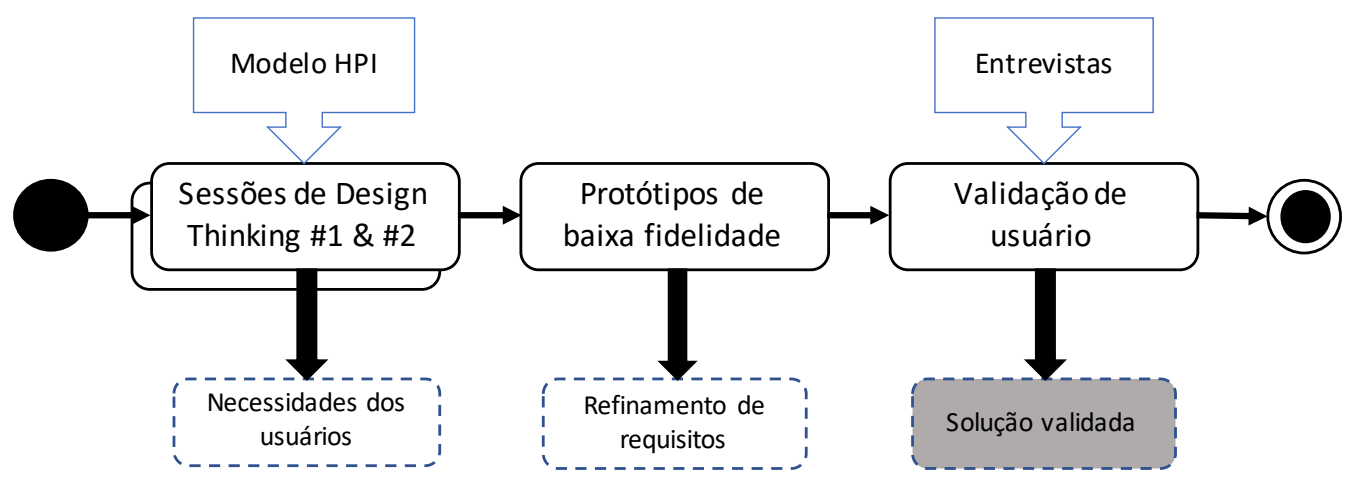

Figura 1. Metodologia da Pesquisa.

As sessões de DT ocorreram com equipes interdisciplinares. O rol de participantes foi composto por dois professores de ciências da computação, dois professores de eventos, um professor da área da saúde, um pesquisador, um profissional de comunicação, cinco estudantes de graduação, sendo quatro do Bacharelado de Sistemas de Informação e um do Tecnólogo em eventos. Os encontros foram realizados em uma sala dedicada no Instituto Federal Farroupilha, unidade de São Borja. Além disso, foram utilizados um quadro, flipcharts, canetas, post-its e papéis disponíveis para manuseio e criação.

Os participantes, após assinatura do Termo de Consentimento e Livre Esclarecido aprovado junto ao Comitê de Ética, foram apresentados à pergunta de pesquisa: "Como seria uma estrutura para promover estudos de desenvolvimento de software por estudantes de graduação?". A Figura 2 mostra um recorte da execução das Sessões de DT.
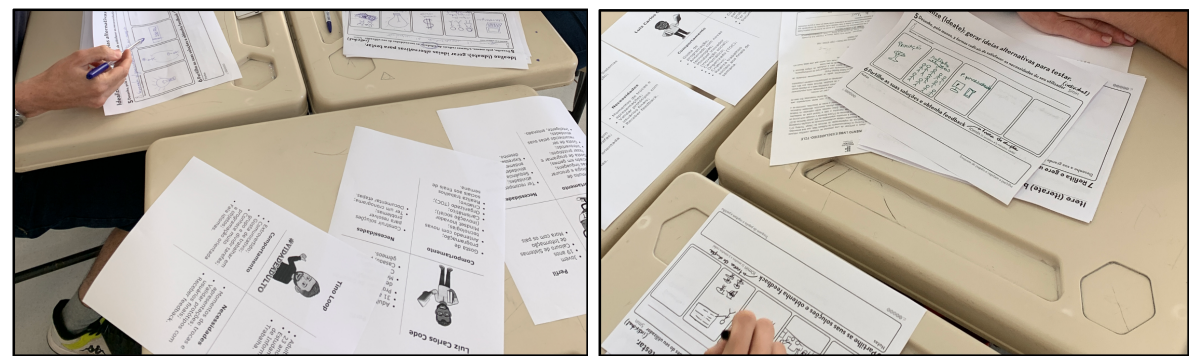

Figura 2. Captura de participação nas sessões de DT.

\subsection{Prototipação}

A primeira versão do gamES é uma solução planejada para Web que fornece recursos para conduzir um processo de DT usando conceitos de gamificação. Os protótipos foram desenvolvidos em nível de baixa fidelidade para refinar os requisitos e um subconjunto deles é mostrado na Figura 3.

Na Figura 3-(a) é representado o acesso do administrador do programa, pelo qual é possível controlar as atividades de uma edição, configurar o comitê de avaliação, ou seja, quem avaliará as soluções, além de inserir uma imagem para identificar a edição atual, bem como o modelo de DT para conduzir as etapas do programa de inovação. Por outro lado, na Figura 3(b), está representada a tela para os participantes (estudantes) organizarem suas atividades. É importante destacar que os itens, tais como as riquezas encontradas e o baú do tesouro, denotam os aspectos de gamificação do gamES. 


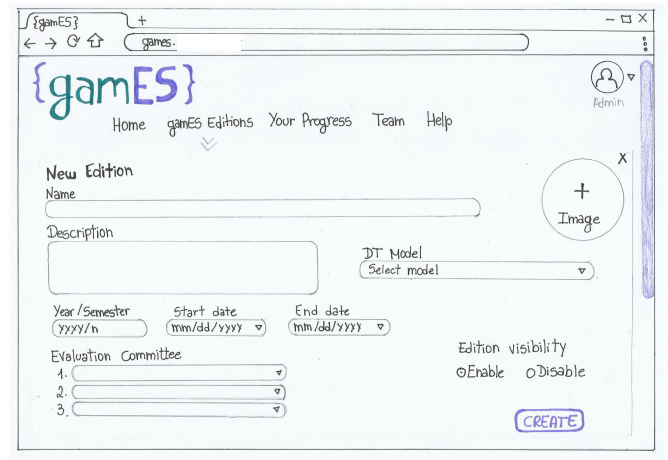

a) Criação da Edição (Versão em Inglês)

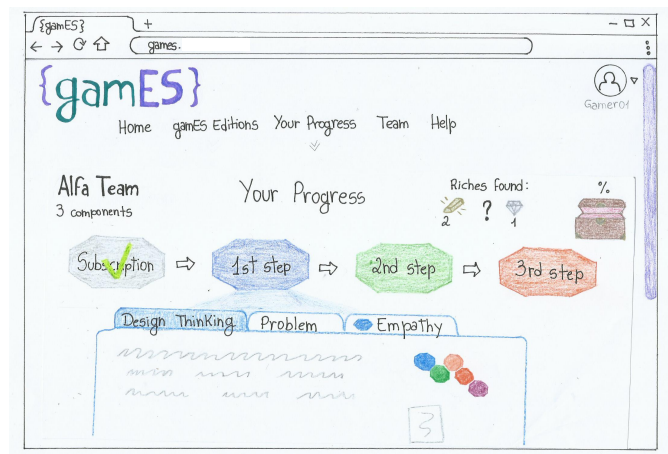

b) Participação equipe (Versão em Inglês)

Figura 3. Protótipos de baixo nível.

Tabela 1. Questões e objetivos - Avaliação da gamES

\begin{tabular}{|c|c|}
\hline Questões & Objetivos \\
\hline $\begin{array}{l}\text { Q1. Você entendeu a proposta e os objetivos do fra- } \\
\text { mework gamES? }\end{array}$ & $\begin{array}{l}\text { Avaliar o entendimento dos entrevistados so- } \\
\text { bre a proposta do projeto. }\end{array}$ \\
\hline $\begin{array}{l}\text { Q2. Sobre a criação de uma edição: quais informações } \\
\text { julga necessário incluir? }\end{array}$ & $\begin{array}{l}\text { Avaliar o entendimento dos entrevistado em } \\
\text { relação aos dados das edições. }\end{array}$ \\
\hline $\begin{array}{l}\text { Q3. Você identificou características de gamificação } \\
\text { nas funções do gamES? }\end{array}$ & $\begin{array}{l}\text { Avaliar a percepção dos entrevistados sobre } \\
\text { aspectos de gamificação. }\end{array}$ \\
\hline $\begin{array}{l}\text { Q4. Em uma escala de } 0 \text { a 5, qual o nível de dificuldade } \\
\text { para usar o framework? }\end{array}$ & $\begin{array}{l}\text { Estabelecer o nível de dificuldade para } \\
\text { utilização do gamES. }\end{array}$ \\
\hline $\begin{array}{l}\text { Q5. Você mudaria alguma funcionalidade no fra- } \\
\text { mework gamES? Se sim, qual(is)? }\end{array}$ & $\begin{array}{l}\text { Avaliar possíveis melhorias no gamES a partir } \\
\text { da visão dos entrevistados. }\end{array}$ \\
\hline
\end{tabular}

\subsection{Validação de usuário}

Com o objetivo de realizar uma primeira avaliação da proposta, realizou-se uma validação de usuário apresentando os protótipos a cinco usuários em potencial, incluindo dois estudantes de graduação, dois professores de ciência da computação e um professor da área de eventos. Este último torna-se importante, visto que se considera a estrutura também como uma ferramenta de gerenciamento de eventos.

Os dados da avaliação foram coletados por meio de entrevistas semiestruturadas, executadas com cada um em um espaço dedicado ao Instituto Federal Farroupilha. A Tabela 1 mostra as perguntas e os respectivos objetivos usados para avaliar a proposta.

\section{Resultados}

Como resultado de uma primeira etapa de pesquisa, executando a sessão dupla de DT e construindo protótipos de fidelidade de baixo nível, são apresentadas as principais características do gamES. A Tabela 2 apresenta uma lista de requisitos e a respectiva ação e usuário, os quais recebem a identificação " $\mathrm{A}$ " para o administrador do sistema e "P" para os recursos disponíveis para o usuário participante. Os recursos disponíveis para $\mathrm{P}$ e $\mathrm{A}$ são representados como "P/A".

Realizaram-se, posteriormente, entrevistas com cinco usuários em potencial, a fim de receber a primeira avaliação da proposta, e, para manter a privacidade dos participantes, estes foram identificados como P1, P2, P3, P4 e P5, como mostrado na Tabela 1. 
Tabela 2. Principais funcionalidades de gamES

\begin{tabular}{lll}
\hline Funcionalidade & Ação & User \\
\hline Gerenciar edições & Criar uma nova edição para o evento & A \\
Registrar modelos de DT & Listar diferentes modelos de DT disponíveis na literatura & A \\
Registrar técnicas associadas a DT & $\begin{array}{l}\text { Registrar as técnicas para serem usadas durante as } \\
\text { sessões de DT }\end{array}$ & \\
Registrar participantes & Realizar o registro individual de alunos interessados em & P \\
& participar & P/A \\
Organizar equipes & Reunir alunos registrados em grupos (equipes) & P/A \\
Exibir equipes & Exibir informações da equipe & A \\
Organizar e moderar inscrições & Auxiliar na organização de equipes ou permissões de & A \\
Definir cronograma & usuário & A \\
Definir tarefas & Criar e manter um cronograma de atividades & - \\
Classificar as equipes & Organizar tarefas para as equipes executarem & Listar as equipes participantes de acordo com suas \\
Calcular pontuação & pontuações & Calcular os pontos das tarefas concluídas pelas equipes \\
Controlar a visualização de tarefas & Organizar as datas em que as tarefas estarão disponíveis & P/A \\
& para as equipes & \\
\hline
\end{tabular}

Inicialmente, perguntas foram feitas aos participantes sobre o entendimento da ferramenta proposta $(\mathrm{Q} 1)$. Eles foram unânimes em relação à compreensão, indicando que os usuários ganhariam prêmios por concluir tarefas e etapas, ajudando a conhecer e usar o Design Thinking - “ [...] [Entendi que] teriam os pontos, para vocêfazer uma etapa de Design Thinking sem saber Design Thinking. Aí, você seguiria os passos e conseguiria fazer o Design Thinking. Seria isso." (P2).

Sobre as informações para criar uma edição $(\mathrm{Q} 2)$, três participantes (P1, P2, P4) sugeriram de as edições do evento de inovação serem norteadas por um tema, um propósito, que fosse dado um desafio a ser atendido, e que a ferramenta auxiliasse na escolha do modelo de DT - "Eu acho que seria interessante para a edição ter um tema. Eu diria que cada edição tem um tema, uma descrição [...]. Para selecionar os modelos de DT, eu não os conheço, então talvez, quando tivesse que selecionar o modelo, tivesse algum tipo de instrução indicando como ele funciona" (P1).

Considerando os elementos de gamificação nos protótipos (Q3), os participantes $(\mathrm{P} 1, \mathrm{P} 2, \mathrm{P} 3, \mathrm{P} 4)$ mencionaram entender a proposta e os elementos, identificando as recompensas e o progresso do processo - "[Entendi] que são essas pontuações que se tornarão ouro. A partir de cada etapa, você realiza as ações. Se você cumprir as tarefas obrigatórias, se fizer mais, ganha mais. Sim, eu entendi.” (P3).

Questionados sobre o nível dificuldade (Q4), os participantes mencionaram que os protótipos e a proposta parecem fáceis de usar, de forma intuitiva - "Para o participante que usa a ferramenta [...] acho muito didático o passo a passo.” (P4).

Por fim, foram indagados sobre os pontos positivos e negativos e sugestões de mudanças (Q5). Os participantes apontaram melhorias para o gamES, como novas funcionalidades, outros tipos de interações e relatórios, gerar uma visão do progresso individual, além do progresso da equipe, com o objetivo de envolver os alunos no uso da estrutura - "Acho que o que mudaria seria uma perspectiva individual, que aqui só tenho uma 
perspectiva de equipe. Gostaria, talvez, em uma visão em equipe, de ver a progressão individual de cada um. Eu acho que seria um fato, uma informação interessante” (P1).

\section{Considerações Finais}

Este artigo apresentou os primeiros resultados de um projeto que visa desenvolver o framework gamES, uma ferramenta para ajudar o processo de ensino e aprendizagem do desenvolvimento de software, que inova ao integrar abordagens de gamificação e Design Thinking. O gamES é composto por um conjunto de recursos, como registrar modelos de DT, registrar técnicas associadas à DT, definir tarefas, classificar equipes, calcular pontuação e, desta forma, orientar o desenvolvimento de software centrado em usuário.

Visando atender os objetivos da pesquisa, apresentou-se o processo metodológico realizado, praticando atividades de DT para entender as necessidades do usuário sobre o tema; prototipação para representar o nível de baixa fidelidade das telas da estrutura; e uma validação antecipada do usuário para avaliar os recursos e as ideias do gamES.

Embora este trabalho possua riscos inerentes a um estudo qualitativo, foram entrevistados professores e estudantes para avaliar diferentes perspectivas do cenário de aprendizagem. Como resultado, mesmo relatando pontos positivos e pontos de melhorias, os participantes da avaliação demonstraram um entendimento adequado da ferramenta.

Os trabalhos futuros para esta pesquisa estão relacionados à profundidade do processo de avaliação, considerando profissionais de outras áreas, professores, alunos de instituições distintas. Além disso, pretende-se aprimorar a prototipação e desenvolver a ferramenta usando uma linguagem de programação moderna e, posteriormente, realizar a validação usando um software funcional.

\section{Agradecimentos}

Este projeto foi financiado parcialmente pelo programa de bolsas PIBIC - Iffarroupilha.

\section{Referências}

Azeem, M. I., Palomba, F., Shi, L., and Wang, Q. (2019). Machine learning techniques for code smell detection: A systematic literature review and meta-analysis. Information and Software Technology, 108:115 - 138.

Blumenfeld, P. C., Soloway, E., Marx, R. W., Krajcik, J. S., Guzdial, M., and Palincsar, A. (1991). Motivating project-based learning: Sustaining the doing, supporting the learning. Educational psychologist, 26(3-4):369-398.

Brum, M. G. and da Cruz, M. E. K. (2017). Gamificação para o ensino de computação na educação básica. In Proceedings of the Workshop sobre Educação em Computação, Rio de Janeiro, Brasil. SBC.

Caroli, P. (2018). Lean Inception: How to Align People and Build the Right Product. Rio de Janeiro, Brasil: Caroli Editora, 160 p.

Desconsi, J. (2012). Design thinking como um conjunto de procedimentos para a geração da inovação: Um estudo de caso do projeta do G3. PhD thesis, Dissertação de Mestrado). Centro Universitário Ritter dos Reis. Porto Alegre, Brasil. 
Dicheva, D., Dichev, C., Agre, G., and Angelova, G. (2015). Gamification in education: A systematic mapping study. Journal of Educational Technology \& Society, 18(3). Douliu City, Taiwan.

Felder, R. M. and Brent, R. (2009). Active learning: An introduction. American Society for Quality higher education brief, 2(4):1-5. Milwaukee, USA.

Ferreira-Oliveira, A., Araújo, A., Fernandes, S. R., and Miguel, I. (2017). Gamification in the workplace: A systematic literature review. In Proceedings of the World Conference on Information Systems and Technologies, pages 283-292. Porto Santo Island, Portugal.

Freitas, R., Peres, S., Fantinato, M., Steinbeck, R., Araújo, U., et al. (2013). Experimenting with design thinking in requirements refinement for a learning management system. In Proceedings of the Brazilian Symposium of Information Systems, pages 182-193. Paraíba, Brasil. SBC.

Hasso Plattner Institute (2010). An introduction to design thinking: In one hour. available in http: / / bit. do/ fxFKZ. accessed on 20/02/2020.

Huotari, K. and Hamari, J. (2017). A definition for gamification: anchoring gamification in the service marketing literature. Electronic Markets, 27(1):21-31.

Kiunsi, D. and Ferwerda, B. (2019). Using a serious game to teach user-centered design. In Proceedings of the International on Intelligent User Interfaces, volume 2327 of CEUR Workshop Proceedings. Los Angeles, USA. ACM.

Krajcik, J. S. and Blumenfeld, P. C. (2005). Project-Based Learning, page 317-334. Cambridge Handbooks in Psychology. Cambridge University Press. Cambridge, Reino Unido.

Poffo, M., Thiry, M., Fernandes, A., and Heemann, C. (2017). Gamificaçao: Agente motivador na aprendizagem de engenharia de software. Proceeding of the Computer on the Beach, pages 110-119. Florianopolis, Brasil.

Pressman, R. and Maxim, B. (2014). Software Engineering: A Practitioner's Approach. McGraw-Hill, New York, USA, 8 edition. 976 p.

Simões, J., Redondo, R., Vilas, A., and Aguiar, A. (2013). Proposta de modelo de referência para aplicação de gamification em ambientes de aprendizagem social. In Atas da Conferência Internacional de TIC na Educação., Minho, Portugal, VIII.

Tomisaki, S., de Souza, A., and Seabra, R. (2016). Mega gp: Aplicando a gamificação no ensino de gerência de projetos. In Anais do XXIV Workshop sobre Educação em Computação, pages 230-239. Porto Alegre, Brasil. SBC.

Vianna, M. (2012). Design thinking: inovação em negócios. Rio de Janeiro, Brasil: MJV Press. $160 \mathrm{p}$.

Weigel, L. (2015). Design thinking to bridge research and concept design. Design Thinking: New Product Development Essentials from the PDMA, pages 59-70.

Werbach, K. and Hunter, D. (2015). The gamification toolkit: dynamics, mechanics, and components for the win. Wharton School Press, Philadelphia, USA. 